\title{
Development of a subspecialty cardiology curriculum for paediatric registrars in Malawi: Implementation of a long-distance hybrid model
}

\author{
Laura Newberry, ${ }^{1}$ Neil Kennedy ${ }^{1}$, Elizabeth A. Greene ${ }^{2}$ \\ 1. Department of Paediatrics and Child Health, College of Medicine, University of Malawi, Blantyre, Malawi
}

2. School of Medicine, University of New Mexico, Albuquerque, New Mexico, USA

Correspondence to: Laura Newberry (lauraanewberry@gmail.com)

\begin{abstract}
Background
Malawi has a high burden of paediatric cardiac disease but a limited number of health providers familiar with these chronic diseases. Given the rising number of Malawian postgraduate paediatric trainees at the University of Malawi College of Medicine, we sought to remedy this lack of basic cardiology training with a long-distance, module-based curriculum that could be utilised independently, as needed, with on-site teaching. We also wished to evaluate the initial modules for utility and improvement in knowledge and confidence in each topic.
\end{abstract}

Abstract

Methods

After an initial site visit to determine curriculum needs, online modules with interactive evaluations and quizzes were developed by a paediatric cardiologist in the United States, in collaboration with paediatric registrar training directors in Malawi. This online interactive curriculum was followed by several site visits to Malawi, by the United States-based paediatric cardiologist, to provide bedside teaching, case-based discussions and hands-on skill training in cardiac ultrasound and electrocardiogram interpretation. Evaluation of the curriculum model included post-module quizzes on cardiac topics as well as registrar self-assessments regarding confidence in content areas.

Results

The average post-module quiz score was $93.6 \%$. Repeat testing with the same questions four months later yielded an average score of $78 \%$, with a range from 60 to $100 \%$. Pre- and post-module registrar self-assessment regarding confidence in content areas showed a substantial gain in knowledge and confidence mean. In their qualitative feedback, registrars noted that the modules were helpful in studying for their certifying examinations, and all four of the registrars sitting Part I of their Malawian and South African paediatric certifying examinations passed.

Conclusions

Our innovative hybrid approach, combining online educational modules with in-person teaching visits, is a useful approach in expanding paediatric cardiology subspecialty education in Malawi.

\section{Introduction}

Malawi has a high burden of paediatric cardiac disease, ranging from congenital heart defects to acquired heart disease. ${ }^{1}$ However, healthcare for children with heart conditions is often inadequate primarily because of the limited availability of healthcare providers familiar with these chronic diseases. In 2008, a small paediatric cardiac clinic with access to echocardiography was established in Blantyre in order to strengthen the care that children with heart disease receive. ${ }^{2}$

At the same time as the opening of the paediatric cardiac clinic, the College of Medicine (COM) of the University of Malawi expanded its postgraduate subspecialty training in paediatrics and child health. Previously, few Malawian graduates of the COM undertook subspecialty training in paediatrics, and most of those who did pursue subspecialty paediatric training did so overseas. ${ }^{3,4}$ As of late 2015, there were seven paediatric trainees in Blantyre and an additional six Malawian trainees completing their rotations in South Africa. ${ }^{4}$ In addition to providing inpatient care of cardiac patients, these trainees also rotate through the various paediatric subspecialty clinics at Queen Elizabeth Central Hospital (QECH) in Blantyre, including the paediatric cardiac clinic. Registrars are also required to know about heart disease for their certifying examinations.

Along with expanding postgraduate programmes, there has been a dramatic increase in North American and European global health paediatric residency partnership programmes within sub-Saharan Africa. However, these programmes tend to focus on providing global health electives for resourcerich learners, while often neglecting the needs of students in developing nations. Few of the US-based institutions with global health programmes have developed reciprocal curricula specifically designed to enhance postgraduate education in developing countries. ${ }^{5}$ However, there are some models demonstrating how an international partnership could adequately address the educational needs of postgraduates in the developing world. Many individuals, organisations, and universities in industrialised countries have worked together with colleagues to establish partnerships in a process known as twinning. ${ }^{6-9}$ Twinning refers to "the establishment of a formal link between a specific department/institution in [a developed country] and a corresponding department/ institution in the developing world." One international partnership in particular, the African Paediatric Fellowship Programme, developed by the Department of Paediatrics and Child Health at the University of Cape Town, provides relevant training for African child health professionals, by Africans, within Africa. ${ }^{10}$ Our Malawian paediatric postgraduate training programme currently sends paediatric registrars to South Africa for one to two years to complete their training. Additional specialised training in certain areas of paediatrics, such as neonatology and oncology, is also available. Even though these partnerships can be valuable, they often do not specifically address how to provide long-distance subspecialty training for students currently training at their home institutions without funding to 
travel to international twin institutions. Given the growing postgraduate training programme in paediatrics, as well as the need for trained healthcare professionals familiar with paediatric cardiac disease, we created a cardiology curriculum for paediatric registrars in Malawi that could be utilised independently, as needed, with on-site and long-distance cardiology consultant teaching through a United Statesbased collaboration. This would provide registrars with the skills needed to evaluate cardiac disease and determine which patients are appropriate to send to outside referral centers.

\section{Methods}

We collaborated on this educational project with the Malawian paediatric registrars at the COM to develop the topics for a long-distance curriculum, which began in April 2013. Prior to the introduction of this formal cardiology curriculum, registrars received limited formal didactic training in cardiology. Within the postgraduate teaching programme, general paediatric consultants within the COM paediatrics department would provide a series of lectures to the registrars regarding a range of paediatric illnesses, including cardiac diseases.

Although these lectures taught the basics of cardiology, they did not always provide an in-depth level of expertise, particularly if the lecturer who taught cardiology was not a cardiologist. Registrars were also informally introduced to both echocardiography and electrocardiography at the bedside. There was no formal training structure to teach and assess these skills. Thus, the goal of the long-distance curriculum was to provide structured, in-depth paediatric cardiology didactic teaching with formal training in necessary cardiology skills, such as echocardiography and electrocardiography. On an initial teaching visit to QECH, in April 2013, our team's paediatric cardiology specialist (EAG, who was on the faculty at Children's Medical Center and George Washington University School of Medicine, both in Washington, D.C.) delivered a series of lectures, as well as teaching sessions in clinics and inpatient settings, to the Malawian registrars. Focus groups with the registrars helped to define additional training topics, as well as the required content for the Malawian paediatric examinations. A major theme that emerged from the focus groups was the registrars' desire to learn about complex congenital heart diseases presenting in infancy. Even though there were no prostaglandins or neonatal cardiac surgical options available at the time, the registrars asked to learn about these disease entities as preparation for when such interventions become available in Malawi in the future. The Malawian registrars also specifically asked for lectures on the pharmacology of cardiac medications, including antiarrhythmic drugs, as they would need to know about them when they spent their final year of training in South Africa and cared for paediatric intensive care patients.

The other major request was for hands-on training in echocardiography. A list of topics was then generated and teaching modules were developed by EAG, in collaboration with cardiology faculty and fellows at Children's National Medical Center. The modules covered the following topics: basic electrocardiography and arrhythmias, the cardiac cycle, left to right shunts, cardiomyopathy, embryology, obstructive lesions, pulmonary hypertension, the cyanotic newborn, rheumatic heart disease, and pharmacology. Each module was developed to be equivalent to a one-hour lecture with a short quiz at the end. Three content questions were formulated on each topic, and self-assessment sections were designed to allow the registrars to evaluate if they had increased their medical knowledge and confidence after each module. The registrars were then given two to four weeks to work on each topic and return the quiz answers, with any questions or comments, to the project's lead paediatric cardiologist or one of the COM registrar training directors.

After the registrars completed the first six modules, EAG conducted a second site visit to QECH. Small group review sessions of each of these modules allowed questions to be answered and any confusing information to be clarified. Special focus was given to the topics that the registrars felt needed more explanation based on their comments and on the preliminary quiz results. The remaining four modules were introduced during this site visit, and the registrars were then given several weeks to complete them and complete the post-module quizzes. Comments on each module and questions about the content were solicited for each module. A third site visit allowed for concentration on the areas of most difficulty, as well as reviews of the principles from the modules with case-based sessions. In particular, the topics with the most concern were repeated in extended teaching sessions on electrocardiogram interpretation, antiarrhythmic medication, and pharmacology. Bedside and clinic teaching was done on each visit, during which echocardiography skills were assessed.

\section{Results}

The results of the post-module quizzes are shown in Table 1. The mean overall module score was $93.6 \%$. The module on cardiomyopathy, congestive heart failure, and pharmacology produced the lowest overall score $(86.7 \%)$. These modules were set up as self-directed learning exercises and the number of registrars completing each module ranged from 2 to 10.

\begin{tabular}{|c|c|}
\hline Module topic & $\begin{array}{l}\text { Answers } \\
\text { Correct/Total (\%) }\end{array}$ \\
\hline 1. Basic Electrocardiogram Interpretation & $21 / 24(87.5)$ \\
\hline 2. Left-to-right Shunts & $28 / 30(93.3)$ \\
\hline 3. Cardiac Cycle & $27 / 27(100)$ \\
\hline 4. Ductal-dependent Cardiac Lesions & $25 / 27(92.6)$ \\
\hline 5. Obstructive Lesions & $25 / 27(92.6)$ \\
\hline 6. Pulmonary Hypertension & $18 / 18(100)$ \\
\hline 7. Cardiac Embryology & $14 / 15(93.3)$ \\
\hline 8. Rheumatic Heart Disease & $6 / 6(100)$ \\
\hline 9. Arrhythmias: Review and Pharmacology & $14 / 15(93.3)$ \\
\hline 10. Cardiomyopathy, Congestive Heart Failure, and Pharmacology & $13 / 15(86.7)$ \\
\hline
\end{tabular}

Four months after the modules were used and tested, repeat content testing was done. Six registrars participated in retesting. Fifteen questions drawn from the original set were selected. The average percent correct was $78 \%$, with a range of 60 to $100 \%$.

Table 2 and Table 3 show the registrars' pre- and post-module self-assessments of content knowledge and confidence in utilising module content in clinical practice, using a fivepoint rating scale. A score of 1 indicated an inexperienced clinician who is just learning about the basic concepts for the first time, and 5 indicated an experienced clinician who is able to teach the basic concepts to a junior trainee. All modules showed an increase in mean self-assessment scores 
of at least 1.0 points; the highest self-assessment mean score increase was 2.0. The registrars had the least confidence in their knowledge of arrhythmias, and the self-assessment scores, both before and after this module, were the lowest compared to the other modules. However, the registrars' performed well on the arrhythmia module, and their knowledge was seen to improve.

Table 2: Pre- and post-module self-assessment of content knowledge Self-assessment of content knowledge

\begin{tabular}{cccc} 
Module & Before $^{\dagger}$ & After $^{\dagger}$ & Increase $^{\dagger}$ \\
\hline 1 & 2.6 & 4.1 & 1.5 \\
2 & 3.0 & 4.7 & 1.7 \\
3 & 2.5 & 4.0 & 1.5 \\
4 & 2.9 & 4.6 & 1.7 \\
5 & 2.4 & 4.4 & 2.0 \\
6 & 2.8 & 4.3 & 1.5 \\
7 & 2.2 & 3.8 & 1.6 \\
8 & 3.5 & 4.5 & 1.0 \\
9 & 1.6 & 2.8 & 1.2 \\
10 & 2.0 & 4.0 & 2.0 \\
\hline Mean & 2.6 & 4.1 & 1.6
\end{tabular}

$\dagger$ Mean score calculated from all self-assessment scores reported by participants on a scale from 1 to 5; a score of 1 indicated a novice clinician who is just learning about the basic concepts for the first time and 5 indicated an experienced clinician who is able to teach the basic concepts to a junior trainee

Table 3: Pre- and post-module self-assessment of utilizing content in clinical practice

\begin{tabular}{cccc}
\hline & \multicolumn{2}{c}{ Self-assessment of confidence in utilising content in practice } & \\
\cline { 2 - 3 } Module & Before $^{\dagger}$ & After $^{\dagger}$ & Increase $^{\dagger}$ \\
\hline 1 & not done & 3.5 & - \\
2 & 2.9 & 4.3 & 1.4 \\
3 & 2.5 & 4.0 & 1.5 \\
4 & 2.7 & 4.4 & 1.7 \\
5 & 2.3 & 4.4 & 2.1 \\
6 & 2.8 & 4.3 & 1.5 \\
7 & 2.2 & 3.6 & 1.4 \\
8 & 4.0 & 4.5 & 0.5 \\
9 & 2.2 & 3.2 & 1.0 \\
10 & 2.6 & 4.2 & 1.6 \\
\hline Mean & 2.7 & 4.0 & 1.4 \\
\hline
\end{tabular}

${ }^{\dagger}$ Mean score calculated from all self-assessment scores reported by participants on a scale from 1 to 5 ; a score of 1 indicated a novice clinician who is just learning about the basic concepts for the first time and 5 indicated an experienced clinician who is able to teach the basic concepts to a junior trainee

Focus groups during the third site visit included discussions of the long-term utility of the modules that had been provided the year before. The registrars reported that they used the modules when studying for their certifying examinations and that the information provided was very helpful and dealt with the content expected of them. There was a $100 \%$ pass rate, among the registrars who completed the modules, on their Malawian and South African Part I paediatric certification examinations.

\section{Discussion}

The initial results of our long-distance hybrid teaching programme of combining online educational modules in conjunction with on-site, in-person teaching visits, suggest that this model can expand paediatric subspecialty education in Malawi. Prior to the introduction of our hybrid cardiology curriculum, cardiology teaching within the postgraduate paediatric training programme occurred informally and was often dependent on the varying expertise and motivations of general paediatricians within the department. The registrars indicated and demonstrated increased knowledge and confidence related to all topics covered in this structured curriculum. Retesting four months after the modules were completed demonstrated retention of the knowledge gained. The registrars have reported that they continued to use the modules on their own, especially to study for their certifying exams. It is too soon to comment on whether our curriculum has had a positive impact on these registrars' preparedness for the final stages of their paediatric training in South Africa, or their success in Part II of their South African certification examinations. We would like to assess this in the future with a follow-up analysis.

Although our numbers were small, we believe the results to be encouraging and demonstrate a sustainable model that can be used for other subspecialty training. The module content was co-developed by the COM Department of Paediatrics in Malawi and a United States-based paediatric cardiology consultant to address necessary clinical content needed in Malawi's low-resource setting.

The overarching goal was to provide a sustainable resource that registrars in Malawi could use to provide clinical care to children with cardiac disease by giving the registrars a resource they could turn to with questions at any time. The cardiology consultant was available via email to answer questions for the registrars regarding the modules or any clinical query. We are in the process of creating an online forum for questions and discussions to further facilitate this process. The modules are currently being used in an open access format for ongoing educational sessions with residents and registrars in both the United States and Malawi. Content will be updated periodically to reflect changing practices and new evidence-based treatments as they become increasingly available in Malawi. Further long-distance teaching sessions are planned in combination with periodic on-site, in-person teaching visits. During these in-person visits, proficiency of echocardiography and electrocardiography skills will also be assessed. Modules will now be developed by the paediatric faculty at the University of Malawi to cover common clinical problems unique to a tropical environment. Topics planned are HIV cardiomyopathy, African-type endocardial fibroelastosis, and rheumatic heart disease, with a focus on the clinical diagnosis and management of each. The COM Department of Paediatrics and Child Health also has a Malawian paediatrician completing his three-year subspecialty training in cardiology, through the College of Medicine of South Africa, at the Steve Biko Academic Hospital in Pretoria. When he returns to Blantyre in 2017, this preliminary programme will benefit from his daily onsite expertise to ensure its future sustainability.

Limitations of our study include that this was a small pilot project in one centre. Our focus was on the feasibility of the development of a long-distance curriculum to cover the requested content and determine if the modules were initially 
useful to the registrars. While we assessed pre- and postmodule self-confidence, we only administered post-module quizzes. To more adequately quantify our achievements, it would have been useful to obtain pre-module objective examination scores before each student attempted the module. There was also variable usage of the modules, or at least completion of the post module quizzes and return of the post-test answers. In-person discussions, e-mails, and questions about the modules suggested that they were being used and appreciated, but these results were not quantified.

\section{Conclusion}

Our innovative hybrid approach combining online educational modules with in-person teaching visits is a useful approach in expanding paediatric cardiology subspecialty education in Malawi.

\section{Acknowledgements}

The authors wish to thank the pediatric cardiology fellows and physicians at Children's National Medical Center for their contributions on the modules listed below. All modules were also edited by Dr. Elizabeth Anne Greene.

The authors also wish to acknowledge the Elda Arce Educational Award at Children's National Medical Center for their support for books and teaching materials and partial support for Dr. Greene's travel to Malawi for her second site visit in 2014.

\section{References}

1. Kennedy N, Miller P, Hoosen E, Cilliers A, Hugo-Hamman C, Brown $\mathrm{S}$, et al. The spectrum of paediatric cardiac disease presenting to an outpatient clinic in Malawi. BMC Res Notes. 2013;6(1):53.

2. Selman AMR, Kennedy N, Borgstein E. G148(P) Defining the burden of paediatric cardiac disease in Malawi - the experience from a tertiary referral centre. Arch Dis Child. 2013;98(Suppl 1):A69.

3. Muula A, Komolafe O. Specialisation patterns of medical graduates, University of Malawi College of Medicine Blantyre. Cent Afr J Med. 2002 Jan $1 ; 48(1)$.

4. Kennedy N, Newberry L. [Postgraduate paediatric training statistics]. Unpublished raw data. University of Malawi College of Medcine and Queen Elizabeth Central Hospital, Blantyre, Malawi; 2015.

5. McKinley DW, Williams SR, Norcini JJ, Anderson MB. International exchange programs and U.S. medical schools. Acad Med. 2008 Oct;83(10 Suppl):S53-7.

6. MacDonagh R, Jiddawi M, Parry V. Twinning: the future for sustainable collaboration. BJU Int. 2002 Mar;89(s1):13-7.

7. Nicoll A, Carter E, Golden B, Robson J, Southall D, Williams T. Personal practice: developing sustainable international partnerships in child health and paediatric care. Arch Dis Child. 2001 Apr 1;84(4):3159.

8. Parry E, Parry V. Training for health care in developing countries: the work of the Tropical Health and Education Trust. Med Educ. 1998 Nov;32(6):630-5.

\begin{tabular}{|c|c|c|c|}
\hline Module & Topic & Fellow & Consultant \\
\hline 1 & $\begin{array}{l}\text { Basic Electrocardiogram } \\
\text { Interpretation }\end{array}$ & Ann Punnoose, MD & E. Anne Greene, MD \\
\hline 2 & Left-to-right Shunts & Peter Dean, MD & Jessica Colyer, MD \\
\hline 3 & Cardiac Cycle & Bradley Clark, MD & Kristin Burns, MD \\
\hline 4 & Ductal-dependent Cardiac Lesions & Jason Patregnani, MD & Russell Cross, MD \\
\hline 5 & Obstructive Lesions & Michael Cunningham, MD & Sarah Clauss, MD \\
\hline 6 & Pulmonary Hypertension & Asha Nair, MD & E. Anne Greene, MD \\
\hline 7 & Cardiac Embryology & & Lowell Frank, MD \\
\hline 8 & Rheumatic Heart Disease & Bradley Clark, MD & Andrea Beaton, MD \\
\hline 9 & $\begin{array}{c}\text { Arrhythmias: Review and } \\
\text { Pharmacology }\end{array}$ & Chris Jordan, MD & E. Anne Greene, MD \\
\hline 10 & $\begin{array}{l}\text { Cardiomyopathy, Congestive Heart } \\
\text { Failure, and Pharmacology }\end{array}$ & Michelle Ploutz, MD & Janet Scheel, MD \\
\hline
\end{tabular}

9. Richards T. Links between doctors across the world bring benefits. BMJ. 2006 Apr 15;332(7546):873.

10. Wilmshurst JM, Morrow B, du Preez A, Githanga D, Kennedy N, Zar HJ, et al. The African pediatric fellowship program: training in Africa for Africans. Pediatrics. 2016 Jan;137(1):36-43. 